\title{
The design and experience of a Regional Intensive Care Unit
}

\author{
J. R. HoYle \\ M.A., F.F.A.R.C.S., D.A. \\ Senior Registrar in Anaesthetics
D. C. ROWE-JONES
M.B., F.R.C.S. \\ Senior Registrar in Surgery
}

Royal Victoria Hospital, Bournemouth

\begin{abstract}
Summary
A 2-year survey has been made of the work of an Intensive Care Unit in a provincial general hospital of 358 beds. We have reviewed from this experience those problems relevant to the establishment of similar units in other parts of the country.

\section{Introduction}

In recent years the Intensive Care Unit has become a well recognized means of care for many urgently and dangerously ill patients. Most of these units have been established at the main teaching or large regional hospitals, often as part of a specialized department and with its particular function chiefly in mind. Indeed, the reports on the subject deal for the most part with this type of unit, to the neglect of the small unit in the provincial general hospitals.

On 27 September 1965, an Intensive Care Unit was started at the Royal Victoria Hospital, Boscombe and our experience here has offered an opportunity for comment on the organization and requirements of such a unit, on the type of case admitted and on the demands such a unit makes on the hospital in general. In all these particulars the needs and purpose of larger or specialized units are not necessarily relevant.
\end{abstract}

\section{Clinical and administrative organization of the unit}

We chose to make a prospective study in which a pro forma was prepared for each patient. In this way our patients were easy to review and relevant information was readily available. This survey covers a 2-year period from 1 January 1966 to 31 December 1967, in which 636 patients of all types rather than just those in respiratory failure were admitted to the unit's four beds. On no occasion have additional beds been made available as this would necessarily lower the standard of individual care. The hospital of 358 beds deals with all types of patients other than cardio-thoracic and neurosurgery within a radius of 20 miles and with a population of 350,000 .

The nursing complement comprises four sisters, four staff nurses, one trainee nurse and four auxiliary nurses. Duties are arranged on the basis of 8-hourly shifts with one sister on duty at all times.

When a patient is transferred to the Intensive Care Unit, he remains in the care of the consultant under whom he was first admitted. In an emergency, the patient admitted directly to the unit is cared for by the surgeon or physician of the day. Daily group ward-rounds have not been possible but consultants in other specialities are available when necessary. One consultant in anaesthetics is responsible for the equipment. The consultant responsible for the patient usually decides when that patient leaves the unit. Exceptionally, a system of arbitration is operated, either by the consultant anaesthetist on call or by the senior surgical or anaesthetic registrars.

The Intensive Care Unit is adjacent to the theatre. There is a recovery room between them to which patients are admitted following surgery. This is staffed by nurses from the unit. There are three advantages in this design. Firstly, economy of nurses is achieved since both the recovery room and the unit are staffed by the same personnel. Secondly, the close proximity of the unit and recovery room to the theatre suite permits close liaison with anaesthetic and surgical staff in the event of an emergency. Finally, surgical patients are easily moved to and from the operating theatre.

The unit has been designed to accommodate seven beds, only four of which have been in use because of shortage of nursing staff. Each bed is supplied with piped oxygen and vacuum, together with a bank of four power sockets and a large notice board for the display of relevant information on each case. Tipping beds are available as necessary, otherwise standard hospital beds and mattresses have proved satisfactory. An angle-poise light is situated on the wall above each bed. Segregation of the sexes was found to be unnecessary. A small area at the end of the room is partitioned off for the preparation of sterile trolleys.

A small room next to the recovery room is equipped as a laboratory.

The unit has the following special equipment:

Ventilators: two East Radcliffe and one Cape. 
Humidifiers: two Hot Water Blower Type, and two Oxygenaire.

Oscilloscopes: four Cardiorater, Type 64.

Electrocardiograph: one Cardiac Recorders Minigraph, Type 4lE.

Defribrillator: one Cardiac Recorders, Type $61 \mathrm{~A}$.

Pacemaker: one Cardiac Recorders, Type 67A.

Oximeter: one Kipp and Zonen Haemoreflector MO 1.

Respirometers: four Wright.

Peak flow meter: one Wright.

Vitalograph.

Carbon dioxide analyser: one Drager pattern; a micro-Astrup blood gas analyser with an oxygen electrode is available in the biochemistry laboratory.

\section{Survey results}

Of the population of England and Wales, $4.3^{\circ}$ 。 is over the age of 75 ; in the Bournemouth and East Dorset Group this figure is $7.2 \%$. This means that there are in Bournemouth 11,250 more persons over the age of 75 than would be found in a typical population of significant size elsewhere. This is reflected in the age spectrum of patients admitted to the Intensive Care Unit, $60 \%$ of whom were over 50 years of age (Fig. 1).

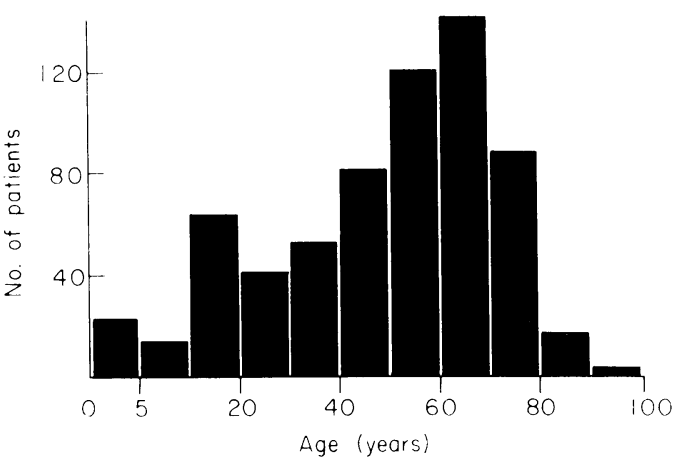

FIG. 1. The age spectrum of patients admitted during the period 1966-68. Sixty per cent of the patients are over 50 years of age.

\section{Diagnostic groups and reasons for admission}

In 2 years, 372 men and 264 women were admitted. iv Fig. 2 shows the types of cases admitted, from which ${ }^{\circ}$ it will be seen that most of them fell into four categories: surgical, coronary thrombosis, traumatic $\vec{c}$ injury and drug overdose. There was no significant difference between the 1st and 2nd years of the survey.

The immediate reason for admission was often t $\overrightarrow{0}$ same in differing diagnostic groups (Fig. 3). Fifo example, unconsciousness might be the reason fer

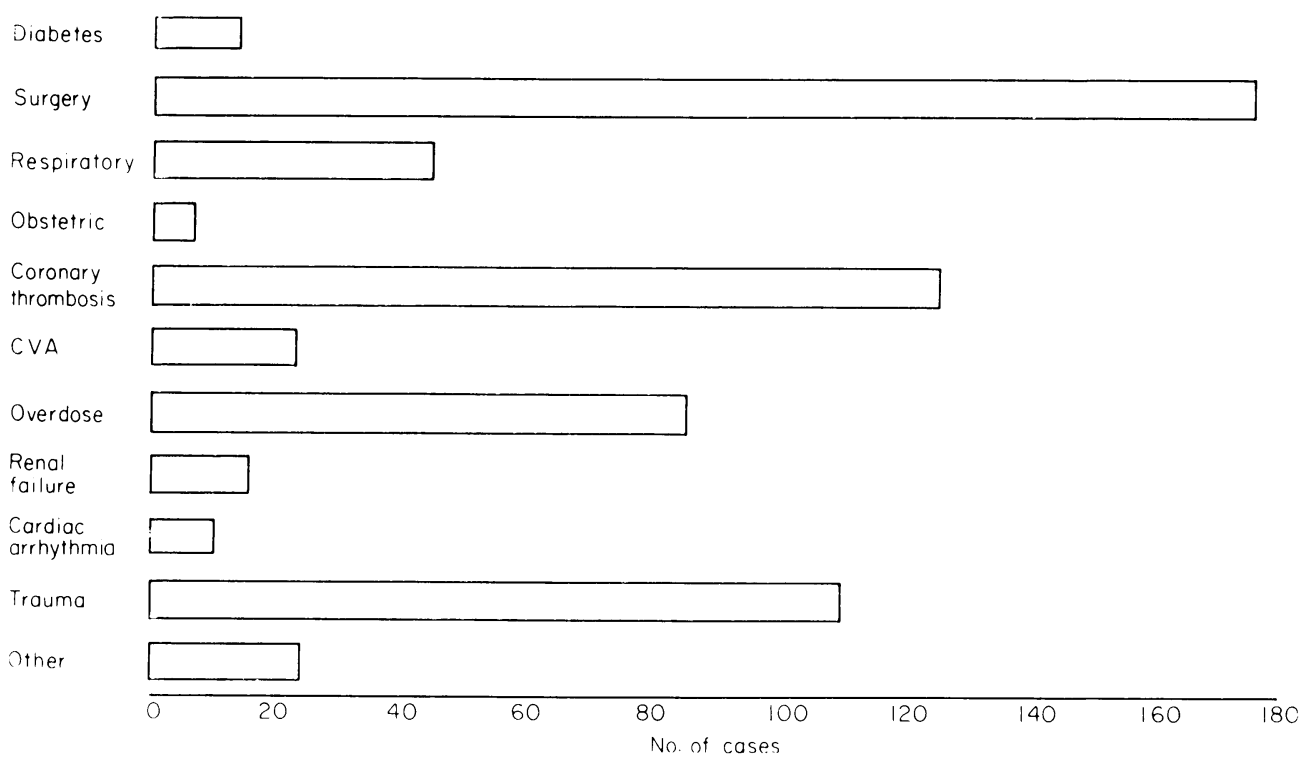

FIG. 2. Diagnostic groups. Seventy-eight per cent of patients admitted were surgical, coronary thrombosis. traumatic injury or drug overdose. 


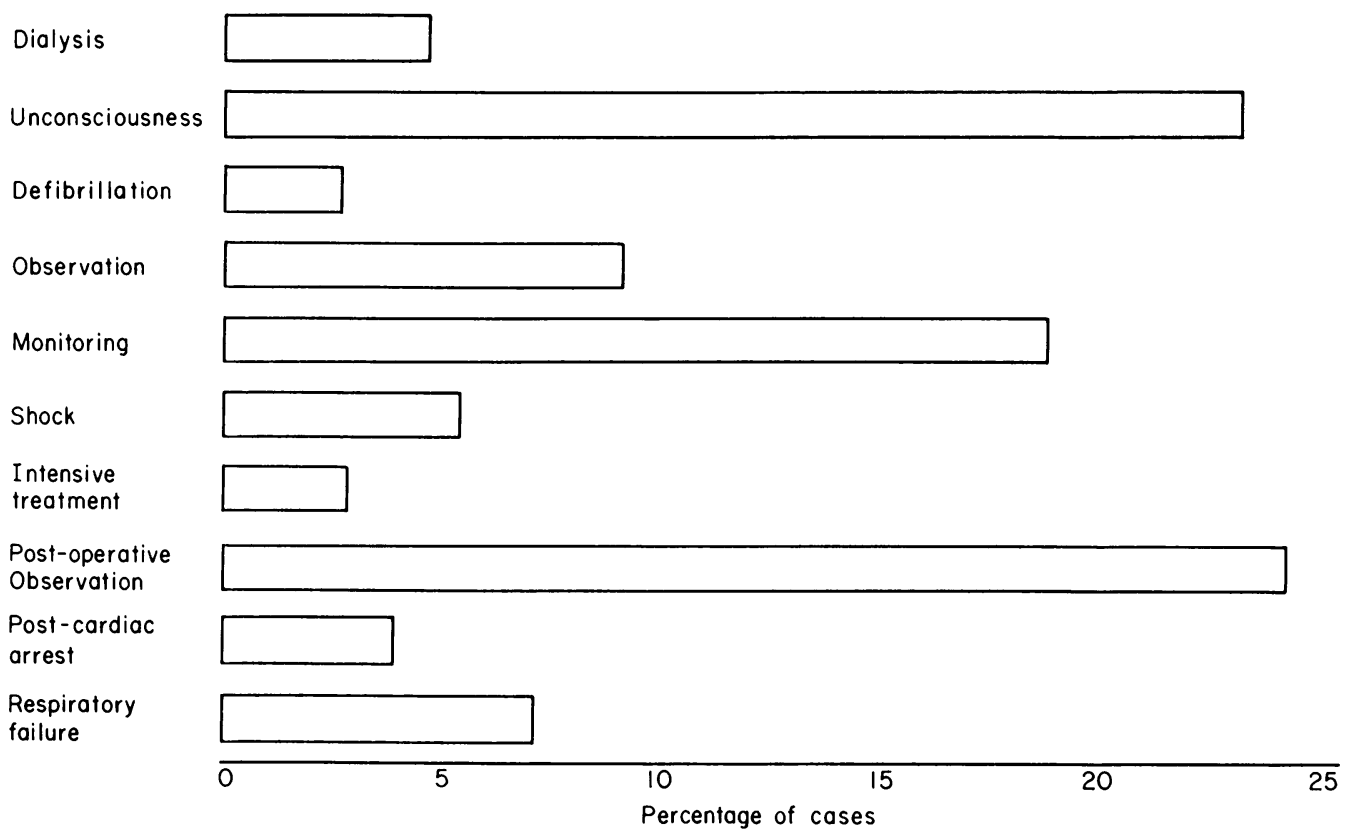

FIG. 3. Reasons for admission. Sixty-five per cent of patients were admitted because of unconsciousness, for post-operative observation or for monitoring.

admission in the diabetic or following a coronary thrombosis, drug overdosage or traumatic injury. The main reasons for admission (Fig. 3) were unconsciousness, post-operative observation or monitoring. These three groups together comprised $65 \%$ of all cases.

\section{Bed occupancy}

The demand each diagnostic group made on the unit was related not only to the number of cases in each group but also and more particularly to the differences in their length of stay. In order to assess this problem the diagnosis and the number of days the bed was occupied was recorded for each patient (Table 1) to give a bed-occupancy figure for each group.

Surgical patients were numerically greater than those in any other diagnostic group. However, in terms of total bed-days, patients with traumatic injury take up much more time. Over the 2-year period, there was an $80.0 \%$ bed occupancy. All four beds were in use on 309 of the 730 days covered in the survey. The average length of stay for patients in all diagnostic groups was 3.6 days. Holidaymakers almost double the local population in the summer months. Surprisingly this has not resulted in an increased demand for beds in the unit.
TABLE 1. Bed occupancy

\begin{tabular}{lccc}
\hline \multirow{2}{*}{$\begin{array}{c}\text { Diagnostic } \\
\text { groups }\end{array}$} & \multicolumn{3}{c}{$1966-68$} \\
\cline { 2 - 4 } & $\begin{array}{c}\text { No. of } \\
\text { patients }\end{array}$ & $\begin{array}{c}\text { Total bed } \\
\text { days }\end{array}$ & $\begin{array}{c}\text { Average stay } \\
\text { per patient }\end{array}$ \\
\hline Surgery & 174 & 611 & $3 \cdot 5$ \\
Trauma & 111 & 689 & $6 \cdot 2$ \\
Coronary & 126 & 359 & $2 \cdot 8$ \\
Overdose & 86 & 186 & $2 \cdot 2$ \\
Respiratory disease & 45 & 191 & $4 \cdot 2$ \\
Cerebrovascular accident & 23 & 91 & $3 \cdot 9$ \\
Renal failure & 16 & 77 & $4 \cdot 8$ \\
Diabetes & 14 & 48 & $3 \cdot 4$ \\
Cardiac arrhythmia & 10 & 25 & $2 \cdot 5$ \\
Obstetrics & 7 & 21 & $3 \cdot 0$ \\
Others & 24 & 53 & $2 \cdot 2$ \\
\hline
\end{tabular}

The demand each diagnostic group made upon beds in the unit is related to the length of stay per patient and not to the numbers admitted in each group.

\section{Nursing and laboratory load}

To discover the demands made upon nursing and laboratory staff by this unit, patients were graded on an arbitrary points system (Tables 2 and 3 ) into one of three categories, either light, medium or heavy. The nursing load was considered to be heavy if the points totalled 7 or over, medium if between 
TABLE 2. The points allocated to various nursing procedures

\begin{tabular}{lc}
\hline \multicolumn{1}{c}{ Nursing procedure } & Points \\
\hline Intravenous infusion & 1 \\
Artificial ventilation & 3 \\
Tracheotomy & 3 \\
Unconsciousness & 3 \\
Wound drainage & 1 \\
Dressings & 1 \\
Fractures & 1 \\
Monitoring & 1 \\
Routine & 1 \\
Machines & 2 \\
Polypharmacy & 2 \\
Central venous pressure & 1 \\
Peritoneal dialysis & 1 \\
\hline
\end{tabular}

TABLE 3. The points allocated to different daily laboratory procedures

\begin{tabular}{lc}
\hline Daily laboratory procedure & Points \\
\hline Blood & 1 \\
Electrolytes and urea & 1 \\
Blood count and haemoglobin & 2 \\
Enzymes & 2 \\
Other & 1 \\
Urine & 1 \\
Culture & \\
Biochemical & 2 \\
Respiratory & 1 \\
Chemical & 1 \\
Bacteriological & 2 \\
Swab culture & 2 \\
Blood volume & \\
Massive blood transfusion & \\
\hline
\end{tabular}

TABLE 4. The percentage of patients in each grade related to nursing and laboratory procedures

\begin{tabular}{lcc}
\hline Grades & $\begin{array}{c}\text { Nursing } \\
\text { procedures } \\
(\%)\end{array}$ & $\begin{array}{c}\text { Laboratory } \\
\text { procedures } \\
(\%)\end{array}$ \\
\hline Light & 50.5 & 69.6 \\
Medium & 31.4 & 17.4 \\
Heavy & 18.1 & 13.0 \\
\hline
\end{tabular}

The $18.1 \%$ of heavy nursing required a standard of nursing not possible in a general ward. The demand for laboratory investigation was light.

4 and 6 and light if 3 or less. The laboratory load was heavy if the points totalled 5 or over, medium if 3-4 and light if 2 or less. Table 4 shows the results. Of heavy nursing, $37 \cdot 1 \%$ involved the care of road traffic accidents of which $46.5 \%$ were unconscious, and this type of case occupied much nursing time. In addition, $30.2 \%$ of these patients had fractures of one or more limbs. It is important to realize that, although $50.5 \%$ of patients in this unit came into the category of 'light nursing', these patients would occupy considerably more of the available time in a general ward. Our $18.1 \%$ of classified as 'heavy' required almost conti active care which would not be possible in a ward at all.

Contrary to what was expected, the dem laboratory investigation was not great since of patients came into the category of 'light' tory load.

During the period of the survey, appros $150 \mathrm{X}$-rays were required per bed per year.

Of patients, $18.4 \%$ required intubation needed tracheotomy and $13.7 \%$ needed Laboratory tests for the diagnosis and mo. of patients with respiratory failure were ne $17.2 \%$ of cases. A 24-hr service for bl analysis is provided by the biochemistry dep and there has never been any delay in al urgent samples. It is felt, therefore, that the $p_{1}$ of the micro-Astrup blood-gas analyser in 1 laboratory is unnecessary.

Cardiac massage was performed in 13 patients with a successful result in $35.9 \%$ cases of trauma where the injury was so st to result ultimately in cardiac arrest, atte resuscitation were uniformly unsuccessfi results of cardiac massage were most succe the post-operative surgical and respiratory This procedure was also well worthwhile in । coronary thrombosis where a success rate 0 : one-third was achieved.

\section{Mortality}

For the year 1966-67, eighty-six of 337 died, a mortality of $25.5 \%$. The following ye: were fifty-nine deaths among 299 pati mortality of $19.7 \%$. Our overall mortality fc years was $22.8 \%$. Related to age, there . significant correlation either in the series as : or among its separate diagnostic groups.

\section{Discussion}

Our survey of the last 2 years' work in the sive Care Unit confirms our opinion of the $v$ such a unit in a general provincial hospita facility for constant surveillance and the imı availability of specialized monitoring and re: tive equipment is of great value to the sevt patient and to the clinician in charge.

In this hospital of 358 beds, serving a pop of 350,000 , the establishment of a four bed In Care Unit has proved to be only just suffici times, patients suitable for admission have be refused and unless the demand for its sper facilities falls, the provision of at least one ex will be necessary. All four beds have been or on 309 days in the past 2 years. In view of th 
likely that if the nursing staff situation permits, another bed will be brought into use in the future. Much of this need was found to arise from the large proportion of unconscious patients admitted. Sometimes, these patients remained for several weeks, when the bed occupancy was total. Often, after initial care, such patients, although unsuitable for the general wards, did not need prolonged intensive care in the unit. With the establishment of an Intensive Care Unit, the severe head injury who before died now survives and becomes a problem of disposal. Proper provision for the care of such patients would dispense with the need for larger Intensive Care Units.

We have found that the nursing complement of four sisters, four staff nurses, one junior nurse and four auxiliary nurses is just adequate to deal with a four-bed unit.
In any hospital with good pathological services, the establishment of a unit such as ours should not create a special problem. Our survey suggests that the main work of a unit in a provincial hospital would be the care of coronary thrombosis, traumatic injury, drug overdosage and the post-operative patient.

Many units have reported a high incidence of cross-infection but in the $2 \frac{1}{2}$ years of this unit this has not been a major problem. This may well be because we have treated very few patients with chronic respiratory disease.

Consultants in all specialities are free to admit their cases to the Intensive Care Unit and retain clinical responsibility for them. We consider this practice to be desirable in maintaining continuity of care. This has not resulted in any problems of administration. 